\title{
Efecto del Calor Aportado y la Cantidad de Capas sobre la Evolución Microestructural en Recargues de Acero Inoxidable Dúplex
}

\author{
Sebastián Zappa ${ }^{1,2}$ (D) , Juan Martinez ${ }^{1,3}$, Hernán Svoboda²,4 \\ ${ }^{1}$ Universidad Nacional de Lomas de Zamora, Facultad de Ingeniería, Buenos Aires, Argentina. \\ ${ }^{2}$ Consejo Nacional de Investigaciones Científicas y Técnicas, Buenos Aires, Argentina. \\ ${ }^{3}$ Instituto de Tecnología Industrial, Buenos Aires, Argentina. \\ ${ }^{4}$ Universidad de Buenos Aires, Facultad de Ingeniería, Buenos Aires, Argentina.
}

Como citá: Zappa S, Martinez J, Svoboda H. Efecto del calor aportado y la cantidad de capas sobre la evolución microestructural en recargues de acero inoxidable dúplex. Soldagem \& Inspeção. 2019;25:e2510. https://doi.org/10.1590/0104-9224/SI25.10

\begin{abstract}
Resumen: Se estudió el efecto de la velocidad de enfriamiento y de la dilución en soldaduras de recubrimiento con un acero inoxidable dúplex, sobre una chapa de acero de bajo contenido de carbono, mediante el proceso de soldadura semiautomático con protección gaseosa. Para estudiar dichos efectos, se soldaron cupones con 0,$6 ; 0,9$ y $1,8 \mathrm{~kJ} / \mathrm{mm}$ (manteniendo constante los parámetros eléctricos y variando la velocidad de soldadura) con una y dos capas. Se midió la composición química, se cuantificó el contenido de fases y se observó la precipitación de compuestos intermetálicos mediante microscopía óptica, electrónica de barrido y difracción de rayos $X$. Los resultados muestran que, en los cupones soldados con una capa, al aumentar el calor aportado, el contenido de ferrita aumenta asociado a la disminución de la dilución. Por otro lado, en los cupones soldados con dos capas (con bajos valores de dilución), el contenido de ferrita disminuye, asociado a la velocidad de enfriamiento y al mayor tiempo disponible para la transformación de ferrita en austenita. El equilibrio microestructural en recargues resistentes a la corrosión con aceros inoxidables dúplex está controlado, principalmente, por el grado de dilución para una capa y por el calor aportado para dos capas.
\end{abstract}

Palabras clave: Aceros inoxidables dúplex; Recargues resistentes a la corrosión; Velocidad de enfriamiento; Dilución; Equilibrio microestructural.

\section{Effect of Heat Input and Number of Passes on Microstructural Evolution of Duplex Stainless Steel Overlay Welds}

\begin{abstract}
The effect of the cooling rate and the dilution in overlay welds with a duplex stainless steel, on a commercial low carbon steel sheet, by means of the semi-automatic welding process with shielding gas was studied. To study the effect of cooling rate and dilution, coupons were welded with $0.6 ; 0.9$ and $1.8 \mathrm{~kJ} / \mathrm{mm}$ (keeping the electrical parameters constant and varying the welding speed) with one and two layers. On the welded specimens, the chemical composition was measured, the phase content was quantified and the precipitation of intermetallic compounds was observed by optical microscopy, scanning electron and X-ray diffraction. The results show that, in the coupons welded with one layer, as the heat input increases, the ferrite content increases associated with the dilution decrease. On the other hand, in coupons welded with two layers (with low dilution values), the ferrite content decreases, associated with the cooling rate and the longer time available for the transformation of ferrite into austenite. The microstructural balance in Corrosion Resistance Alloy Overlay Welding with duplex stainless steels is controlled, mainly, by the degree of dilution for one layer and the heat provided for two layers.
\end{abstract}

Key-words: Duplex stainless steels; Corrosion resistance alloy overlay; Cooling speed; Dilution; Microstructural balance.

\section{Introducción}

Los aceros inoxidables dúplex (DSS) poseen las características necesarias que debe tener un material para ser utilizado como recubrimiento: son soldables, tienen adecuadas propiedades mecánicas y una alta resistencia a la corrosión localizada [1]. Estos materiales poseen una microestructura balanceada constituida en ferrita (F) y austenita (A), la cual optimiza sus propiedades [2]. Durante el proceso de soldadura estos aceros solidifican en un modo completamente ferrítico y el equilibrio microestructural se genera por la transformación de F en A durante el enfriamiento en estado sólido. La naturaleza de dicha transformación dependerá de la composición química y de la velocidad de enfriamiento [3]. Debido a su alto nivel de aleación, 
estos materiales son susceptibles a la precipitación de fases secundarias durante la exposición a elevadas temperaturas, las cuales pueden afectar tanto su comportamiento frente a la corrosión como sus propiedades mecánicas [4].

En los últimos años, se ha desarrollado el concepto de Corrosion Resistance Alloy Overlay Welding (CRAOW) en numerosas industrias tales como las químicas, petroquímicas y, fundamentalmente, del gas y petróleo. El mismo, es un proceso en el cual una delgada capa de un material resistente a la corrosión, es agregada sobre una pieza de acero al carbono o de baja aleación por soldadura [5]. De esta manera, es posible la fabricación y/o la reparación de elementos o componentes de la ingeniería con una vida útil mayor (pieza en servicio) a un menor costo [6]. Existen varios métodos para recubrir materiales (sustrato). Entre ellos, la soldadura por arco eléctrico es la más empleada por una cuestión económica. En este sentido, el proceso de soldadura semiautomático con protección gaseosa y electrodo consumible tubular relleno de elementos generadores de escoria (FCAW) es el proceso de soldadura mayormente aceptado por la industria en América Latina, especialmente por su mayor productividad y la facilidad de realizar soldaduras en toda posición [7].

En los procesos de soldadura por arco eléctrico, ciertos parámetros controlan el calor aportado $Q=(T x C) / V s$, siendo: $Q$ : calor aportado $(\mathrm{kJ} / \mathrm{mm}), T$ : Tensión(V), $C$ : Corriente (A), $V s$ : Velocidad de soldadura (mm/s). El mismo, determina la velocidad de enfriamiento y la dilución. Ambos factores modifican la composición química, el contenido de fases y la precipitación de compuestos intermetálicos en soldaduras de acero inoxidable dúplex.

El calor aportado empleado durante el proceso de soldadura, modifica la velocidad de enfriamiento y el tiempo de transformación de F en A durante la solidificación [3]. En este sentido, soldaduras con bajo calor aportado presentan una mayor velocidad de enfriamiento (menor tiempo de transformación en estado sólido de $\mathrm{F}$ en A), generando un menor contenido de $A$. De manera contraria, soldaduras con alto calor aportado presentan una menor velocidad de enfriamiento (mayor tiempo de transformación en estado sólido de $\mathrm{F}$ en $\mathrm{A}$ ) generando un mayor contenido de $\mathrm{A}$ [8]. En esta última instancia se da lugar a fenómenos de difusión que podrían promover la precipitación de segundas fases tales como fase Sigma $(\sigma)$, Chi $(\chi)$, etc.

En relación a la evaluación de la dilución en depósitos de soldadura, en general se presentan dos criterios: dilución geométrica y dilución química. La dilución geométrica, generalmente es determinada mediante la relación entre el área del metal base fundido $(B)$ respecto el área total del metal de soldadura $(A+B)$ conforme a la Ecuación $1[9,10]$ :

$D=\frac{B}{A+B} x 100$

De la misma manera, la dilución química queda definida en términos del cambio de la composición química del material de aporte, en la pileta líquida, mediante la mezcla con el metal base. La dilución puede ser determinada mediante la Ecuación 2 [11]:

$D=\frac{C_{m s}-C_{m a}}{C_{m b}-C_{m a}}$

donde $D$ representa la dilución; $C_{m s}$ es la concentración de algún elemento en el metal de soldadura; $C_{m a}$ es la concentración de algún elemento en el material de aporte y $C_{m b}$ es la concentración de algún elemento en el metal base.

El cromo equivalente $\left(\mathrm{Cr}_{\text {eq }}\right)$ representa la influencia del $\mathrm{Cr}$ y de otros elementos alfágenos para estabilizar la $\mathrm{F}$. De la misma manera el níquel equivalente ( $\mathrm{Ni}_{\text {eq }}$ ) representa la influencia del $\mathrm{Ni}$ y de otros elementos gammágenos para estabilizar la A. Por un lado, la dilución reduce los elementos de aleación $(\mathrm{Cr}, \mathrm{Ni}, \mathrm{Mo}$, fundamentalmente) y aumenta el contenido de $C$ del recubrimiento, ya que el contenido de $C$ del material del recubrimiento es muy inferior respecto al contenido de $C$ del sustrato o material base. El efecto de la composición química sobre la microestructura queda determinado por la relación entre $\mathrm{Cr}_{\text {eq }} / \mathrm{Ni}_{\text {eq }}[8,11]$.

En este sentido, la composición química, el balance de fases de A y de F y, por ende, las propiedades finales de CRAOW están fuertemente influenciadas por la velocidad de enfriamiento y la dilución con el metal base alcanzada durante el proceso de soldadura. En este sentido, es aún necesaria una mayor cantidad de trabajo a fin de lograr una mejor comprensión de la influencia de los parámetros del procedimiento sobre microestructura resultante y la composición química de los recargues.

La composición química resultante de los recargue definirá la resistencia a la corrosión localizada de los mismos. Um método comúnmente utilizado para estimar la resistencia a la corrosión localizada (incluido la corrosión por picado y la corrosión intergranular) en aceros inoxidables es el uso del Número Equivalente de Resistencia al Picado, PREN (Pitting Resistance Equivalent Number). Generalmente, un alto PREN representa una alta resistencia a la corrosión localizada. EI PREN se define a partir de tres de los elementos más relevantes en relación a la resistencia a la corrosión ( $\mathrm{Cr}$, Mo y N), de acuerdo a la Ecuación $3[12,13]$ :

PREN $=\% C r+3,3 \% M o+16 \% \mathrm{~N}$

Sin embargo, otros autores utilizan otra expresión del PREN que tiene en cuenta el efecto perjudicial del Mn [14], de acuerdo a la Ecuación 4: 
Por otro lado, se sabe que el balance microestructural de ferrita y de austenita también tiene influencia en el comportamiento a la corrosión [15]. Un desequilibrio microestructural podría generar la perdida de la resistencia a la corrosión.

En los últimos años, la literatura [5-7] ha abordado el tema de CRAOW con consumibles DSS, evidenciando una fuerte demanda de conocimiento científico-tecnológico. Sin embargo, dichos trabajos fueron desarrollados con aceros inoxidables superdúplex (de mayor costo por su mayor nivel de aleación) y mediante el empleo de diferentes procesos de soldadura. Hay poca información respecto al estudio del CRAOW con consumibles FCAW de acero inoxidable dúplex modificando el calor aportado del proceso de soldadura y la cantidad de capas de recargue.

La economía del CRAOW se verá fuertemente afectada por la velocidad de deposición y el número de capas necesarias para alcanzar los requerimientos.

El objetivo de este trabajo fue el de estudiar el efecto del calor aportado (a través de la variación de la velocidad de soldadura) y de la cantidad de capas sobre la evolución microestructural de recargues de un acero inoxidable dúplex, con el fin de optimizar el procedimiento de soldadura, de forma que se garantice una microestructura balanceada, libre de precipitados perniciosos, disminuyendo los tiempos y costos de soldadura (mayor velocidad de soldadura y menor cantidad de capas).

\section{Procedimiento Experimental}

\subsection{Soldadura}

Para cumplimentar con los objetivos del presente trabajo, se soldaron seis cupones CRAOW mediante el proceso de soldadura semiautomático con protección gaseosa. Por un lado, para generar diferentes calores aportados y evidenciar el efecto de la velocidad de enfriamiento, se utilizaron los mismos parámetros eléctricos de soldadura modificando la velocidad de avance, para obtener tres valores de calor aportado $(0,6 ; 0,9$ y $1,8 \mathrm{~kJ} / \mathrm{mm})$. El rango de los calores estudiados, estuvo asociado a emplear parámetros de soldadura estables (determinados a partir de pruebas preliminares) y que no excedan ciertos valores ya que, en estos materiales, no se recomienda superar los $2,5 \mathrm{~kJ} / \mathrm{mm}$ [10]. Por otro lado, para hacer evidente el efecto de la dilución se soldaron cupones con una y dos capas. En todos los casos, en la primera capa se soldaron 5 cordones, mientras que en la segunda 4. El consumible empleado fue un alambre tubular con relleno de elementos fundentes, del tipo FCAW, de 1,2 mm de diámetro que deposita un acero inoxidable dúplex. La posición de soldadura fue plana (1G) y los cordones del recargue fueron soldados en forma mecanizada para controlar la velocidad de avance y mantenerla constante. De acuerdo a las recomendaciones del fabricante del consumible [16], el gas de protección empleado fue una mezcla de Argón con un $20 \%$ de $\mathrm{CO}_{2}$ y un caudal de $18 \mathrm{~L} / \mathrm{min}$. La distancia entre el pico de contacto y la pieza, en todos los casos, fue de $20 \mathrm{~mm}$. Las chapas base utilizadas como sustrato fueron de acero comercial de bajo carbono de $250 \times 75 \times 9,5 \mathrm{~mm}$ (largo $\times$ ancho $\times$ espesor). La Figura $1 \mathrm{muestra}$ un esquema y una fotografía del proceso de soldadura.

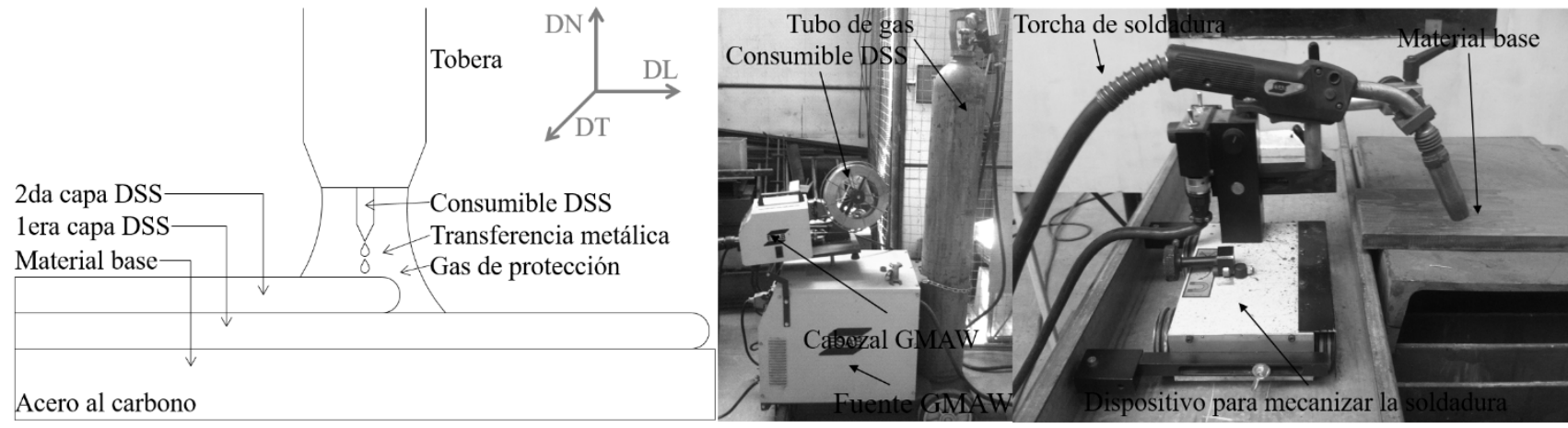

Figura 1. Esquema y fotografía del proceso de soldadura. DN = dirección normal; DL = dirección longitudinal; DT = dirección transversal.

La Tabla 1 muestra la composición química de la chapa base, determinado por espectrometría de emisión óptica, y del metal de aporte puro del consumible utilizado reportado por el fabricante [16]. La Tabla 2 muestra la identificación de cada cupón en la primer coluna: el primer dígito, letra D, corresponde al material de aporte utilizado (DSS), el segundo dígito, números 1 o 2, corresponden a la cantidad de capas del racargue ( 1 o 2 capas) y el tercer dígito, corresponde al niver de aporte térmico (alto: $A$, medio: $M$ y bajo: $B$ ). 
Tabla 1. Composición química del material base y consumible utilizado.

\begin{tabular}{|c|c|c|c|c|c|c|c|}
\hline Material & $\begin{array}{c}C \\
(\%)\end{array}$ & $\begin{array}{c}\mathrm{Si} \\
(\%)\end{array}$ & $\begin{array}{l}\mathrm{Mn} \\
(\%)\end{array}$ & $\begin{array}{c}\mathrm{Cr} \\
(\%)\end{array}$ & $\begin{array}{c}\mathrm{Ni} \\
(\%)\end{array}$ & $\begin{array}{l}\text { Mo } \\
\text { (\%) }\end{array}$ & $\begin{array}{c}N \\
\text { (\%) }\end{array}$ \\
\hline Material base & 0,10 & 0,2 & 0,58 & - & - & - & - \\
\hline Consumible DSS & 0,03 & 0,8 & 0,90 & 22,7 & 9,1 & 3,2 & 0,13 \\
\hline
\end{tabular}

Nota: Valores expresados en porcentaje en peso.

Tabla 2. Identificación y parámetros de soldadura.

\begin{tabular}{|c|c|c|c|c|}
\hline Cupón & $\begin{array}{l}\text { Tensión } \\
\text { (V) }\end{array}$ & $\begin{array}{l}\text { Corriente } \\
\text { (A) }\end{array}$ & $\begin{array}{l}\text { Velocidad de soldadura } \\
\qquad(\mathrm{mm} / \mathrm{s})\end{array}$ & $\begin{array}{l}\text { Calor aportado } \\
(\mathrm{kJ} / \mathrm{mm})\end{array}$ \\
\hline D1B & 28 & 196 & 9,7 & 0,6 \\
\hline D1M & 28 & 195 & 6,4 & 0,9 \\
\hline D1A & 28 & 200 & 3,1 & 1,8 \\
\hline D2B & 28 & 195 & 9,7 & 0,6 \\
\hline D2M & 28 & 195 & 6,4 & 0,9 \\
\hline $\mathrm{D} 2 \mathrm{~A}$ & 28 & 200 & 3,1 & 1,8 \\
\hline
\end{tabular}

Nota: $Q=(T x C) / V s ; \mathrm{Q}=$ calor aportado; $\mathrm{T}=$ Tensión; $\mathrm{C}=$ Corriente; $\mathrm{Vs}=$ Velocidad de soldadura.

\subsection{Caracterización macroestructural y dilución geométrica}

De cada cupón soldado, se extrajeron varios cortes transversales para la caracterización macroestructural. Se determinaron las características geométricas de los recargues (alto, ancho y penetración) y se calculó la dilución geométrica [1,8] de los mismos, de acuerdo a la Ecuación 1 y lo observado en la Figura 2, donde $B$ es el área del metal base fundido y $A$ es el área representativa del material de aporte.

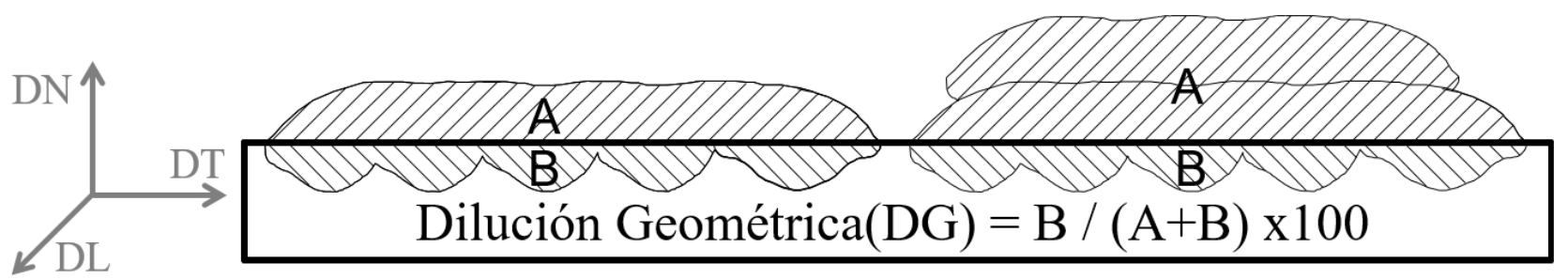

Figura 2. Determinación de la Dilución Geométrica de los recargues. DN = dirección normal; $\mathrm{DL}=$ dirección longitudinal; $\mathrm{DT}=$ dirección transversal.

\subsection{Caracterización microestructural, dilución química y microdureza}

Se caracterizó la microestructura mediante microscopías óptica (LM) y electrónica de barrido (SEM) realizadas sobre cortes transversales de los depósitos de soldadura. Las superficies sometidas a evaluación fueron preparadas mediante desbaste y pulido hasta pasta de diamante de 1 micrón. El reactivo utilizado para revelar y colorear la microestructura fue Beraha (80 $\mathrm{mL}$ $\mathrm{H}_{2} \mathrm{O}+20 \mathrm{~mL} \mathrm{HCl}+1 \mathrm{~g} \mathrm{~K}_{2} \mathrm{~S}_{2} \mathrm{O}_{5}$ ) [17] con un tiempo de exposición inferior a los 10 segundos.

Asimismo, sobre los cortes transversales mencionados se midió, de manera semicuantitativa, la composición química en cada uno de los cordones depositados, mediante la técnica EDS (Energy Dispersive X-ray Spectroscopy) con el fin de estudiar la evolución de la dilución química desde el cordón 1 hasta el cordón 5 en los cupones de una capa y desde el cordón 1 hasta el cordón 4 (segunda capa) en los cupones soldados de dos capas. Por otro lado, en la superficie de los recargues se midió la composición química por espectrometría de emisión óptica (OES) para determinar la dilución química "general” de los recargues. El cálculo de la dilución reportado, fue el promedio de las mediciones de los elementos: $\mathrm{Cr}$, Ni y Mo. Los contenidos de $\mathrm{C}$ y de $\mathrm{N}$ se estimaron mediante lo reportado en el análisis químico del consumible provisto por el fabricante [16] y el porcentaje de dilución, como lo reportan otros autores [18].

Para predecir la microestructura en la superficie de los cupones soldados, teniendo en cuenta la composición química y el porcentaje de dilución, se utilizó el diagrama Schaeffler y el WRC 1992, como lo indica la literatura [19-22].

Sobre la superficie de los cupones se realizó la cuantificación microestructural de ferrita y austenita por medio de metalografía color a 500X y con 40 imágenes para cada condición, mediante el empleo de un software de análisis de imágenes.

Para estudiar con mayor detalle la microestructura final y buscar la presencia de segundas fases o precipitados, sobre la superficie de los recubrimientos se realizaron mediciones de Difracción de Rayos X con radiación de Cu $(\lambda=1,5408 \AA$ ). Para 
maximizar la calidad de los difractogramas y poder identificar la precipitación de segundas fases de bajos porcentajes en volumen, se ajustaron los parámetros de los ensayos de difracción (acotado ángulo de barrido, bajo paso y velocidad de barrido), en un rango de 42 a $47^{\circ}$ ( 2 theta) con un paso de $0,05^{\circ}$ a una velocidad de barrido de $0,5^{\circ} / \mathrm{min}$.

Finalmente, se midieron 20 valores de dureza Vickers con $1 \mathrm{~kg}$ de carga (HV1) sobre las superficies de los cupones, reportándose los valores promedios de los mismos.

\section{Resultados y Discusión}

\subsection{Macrografía y dilución geométrica}

La Figura 3 muestra imágenes de los cortes transversales de los recargues soldados. En las mismas, se observa la chapa base, la zona afectada por el calor y el metal de soldadura conformando el recubrimiento (una o dos capas).

De acuerdo con la literatura [23], la geometría (tamaño) del cordón es directamente proporcional al calor aportado. Es decir, al aumentar el calor aportado aumentan las características geométricas del cordón de soldadura. En la Tabla 3 se presentan los valores de las dimensiones de los cupones: ancho, alto y penetración medidas sobre cada uno de los recubrimientos. Además, se presentan los valores de las áreas del metal base fundido (B) y total ( $A+B)$ para determinar la dilución geométrica, de acuerdo a la Figura 2. La Figura 4 muestra la evolución de las características geométricas y la dilución geométrica en función al calor aportado.

Tabla 3. Dilución geométrica.

\begin{tabular}{ccccccc}
\hline Cupón & $\begin{array}{c}\text { Penetración } \\
(\mathbf{m m})\end{array}$ & $\begin{array}{c}\text { Ancho } \\
(\mathbf{m m})\end{array}$ & $\begin{array}{c}\text { Alto } \\
(\mathbf{m m})\end{array}$ & $\begin{array}{c}\text { B } \\
\left(\mathbf{m m}^{2}\right)\end{array}$ & $\begin{array}{c}\text { A+B } \\
\left(\mathbf{m m}^{2}\right)\end{array}$ & $\begin{array}{c}\text { Dilución geométrica } \\
(\%)\end{array}$ \\
D1B & 1,1 & 30,8 & 3,6 & 19,2 & 103,7 & 19 \\
D1M & 1,2 & 37,9 & 4,6 & 25,3 & 159,9 & 16 \\
D1A & 1,0 & 54,0 & 4,9 & 27,5 & 234,7 & 12 \\
D2B & 1,0 & 29,5 & 6,5 & 18,5 & 171,8 & 11 \\
D2M & 1,2 & 38,2 & 7,6 & 24,0 & 269,0 & 9 \\
D2A & 1,1 & 52,8 & 10,2 & 35,9 & 422,1 & 8 \\
\hline
\end{tabular}
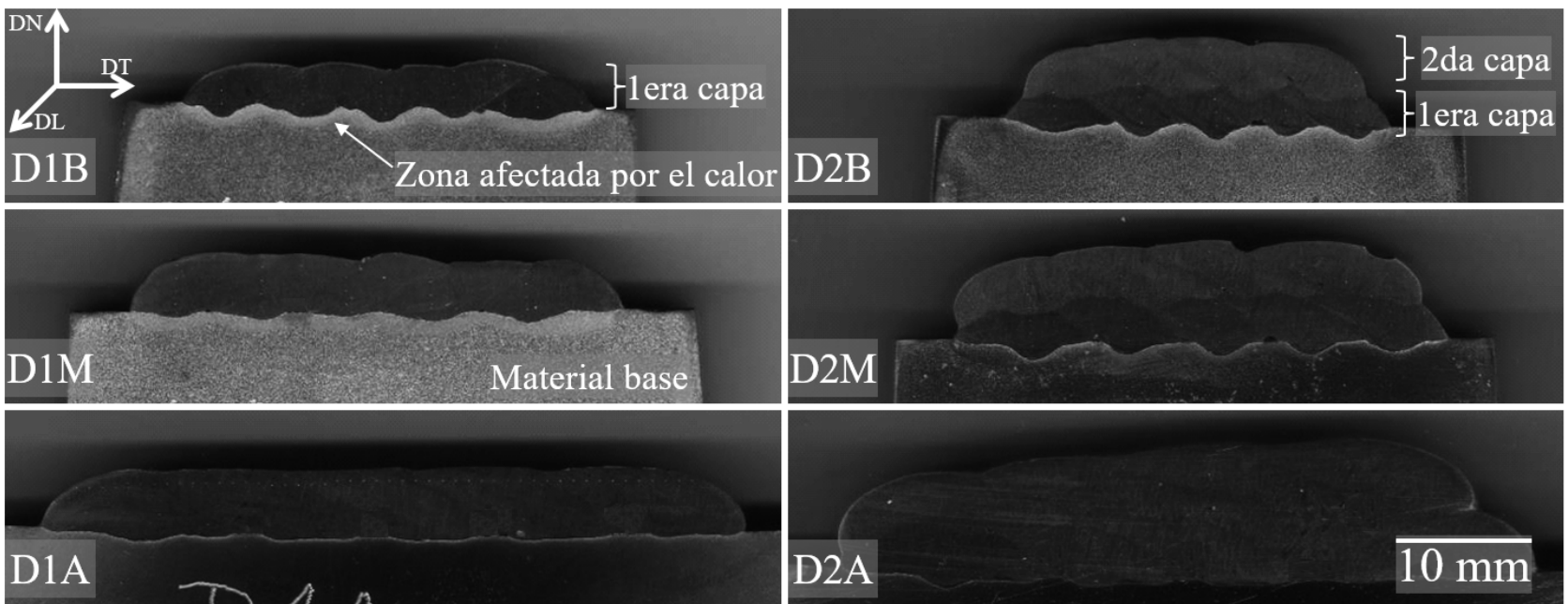

Figura 3. Cortes transversales de los recargues. $\mathrm{DN}=$ dirección normal; $\mathrm{DL}=$ dirección longitudinal; $\mathrm{DT}=$ dirección transversal. 


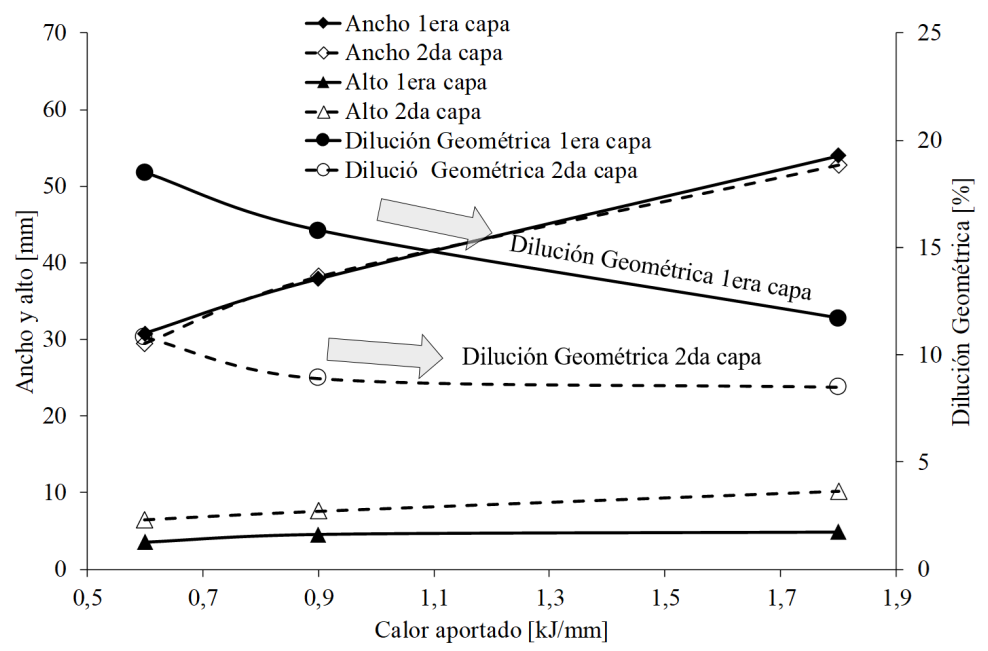

Figura 4. Penetración, ancho, alto y dilución geométrica.

Como puede observarse en la Tabla 3 y en la Figura 4, el aumento del calor aportado generó un aumento en todos los parámetros geométricos del recubrimiento, que se manifiesta en un aumento del área de sección transversal de los cupones. El ancho y la altura del revestimiento aumentaron en la medida que aumentó el calor aportado (controlado por la velocidad de soldadura). Estos fenómenos están asociados a que a medida que la velocidad de soldadura disminuye, el calor aportado por unidad de longitud aumenta, generando un aumento en el ancho y la altura del material depositado [23]. Por otro lado, no se observaron variaciones en la penetración al disminuir la velocidad de soldadura. La dilución geométrica osciló, aproximadamente, entre un 9 y $20 \%$ para las diferentes condiciones de soldadura. En este sentido, el hecho de que la dilución se haya encontrado entre estos valores es un hecho relevante, dada la importancia de la composición química en la formación de las fases microestructurales [8] y en las propiedades superficiales finales del recubrimiento. El porcentaje de dilución (metal base fundido en la pileta líquida) disminuyó con el aumento del calor aportado y de una a dos capas. Este hecho podría estar asociado a que al aumentar el calor aportado y/o el número de capas del recargue, se entregan mayores volúmenes de material aportado respecto al volumen del material base fundido, dando como resultado disminución de la dilución geométrica. Dicho efecto está claramente observado en la Tabla 3 donde se presentan los valores de las áreas del metal de soldadura y del área total de cada recargue que definen la dilución geométrica conforme a la Ecuación 1 . Sin embargo, debe tenerse en cuenta que, si el calor aportado aumenta a través del aumento de la corriente, el resultado de la dilución podría ser diferente.

\subsection{Dilución química}

La Figura 5 muestra la dilución obtenida en los 5 cordones, para los cupones de una capa y en los 4 cordones, para los cupones de dos capas.

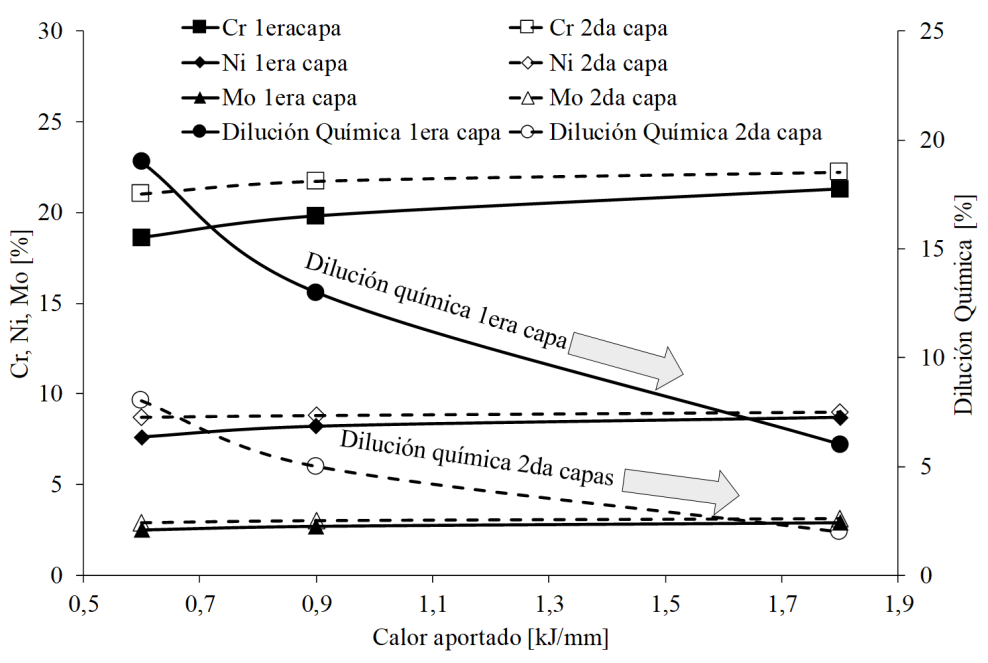

Figura 5. Dilución entre cordones para una y dos capas. 
La Figura anterior se puede observar que la dilución disminuye hasta el cuarto cordón, a partir del cual se estabiliza para las probetas soldadas con una capa. Asimismo, para el caso de la segunda capa en el tercer cordón se alcanza la estabilidad de los valores de dilución. Esto es un dato relevante ya que considerar la microestructura obtenida en las primeras zonas de los cupones podría generar errores, por ser una zona de transición. Los resultados presentados en este trabajo corresponden a lo observado en los últimos cordones (4-5 para una capa y 3-4 para dos capas).

La Tabla 4 muestra la composición química superficial de los recargues y el cálculo de la dilución química, el PREN y el contenido de ferrita. Las Figuras 6.a y 6.b muestran la evolución de la composición y la dilución química al aumentar el calor aportado para una y dos capas, respectivamente.

Tabla 4. Composición y dilución química.

\begin{tabular}{|c|c|c|c|c|c|}
\hline Cupón & $\begin{array}{l}\mathrm{Cr} \\
(\%)\end{array}$ & $\begin{array}{l}\mathrm{Ni} \\
\text { (\%) }\end{array}$ & $\begin{array}{l}\text { Mo } \\
\text { (\%) }\end{array}$ & Dilución química (\%) & PREN \\
\hline D1B & 18,6 & 7,6 & 2,5 & 19 & 28,9 \\
\hline D1M & 19,8 & 8,2 & 2,7 & 13 & 31,1 \\
\hline $\mathrm{D} 1 \mathrm{~A}$ & 21,3 & 8,7 & 2,9 & 6 & 33,7 \\
\hline D2B & 21,0 & 8,7 & 2,9 & 8 & 33,1 \\
\hline $\mathrm{D} 2 \mathrm{M}$ & 21,7 & 8,8 & 3,0 & 5 & 34,1 \\
\hline $\mathrm{D} 2 \mathrm{~A}$ & 22,2 & 9,0 & 3,1 & 2 & 35,2 \\
\hline
\end{tabular}

PREN $=\% C r+3,3 \% M o+30 \% N-M n$ (Ecuación 4).
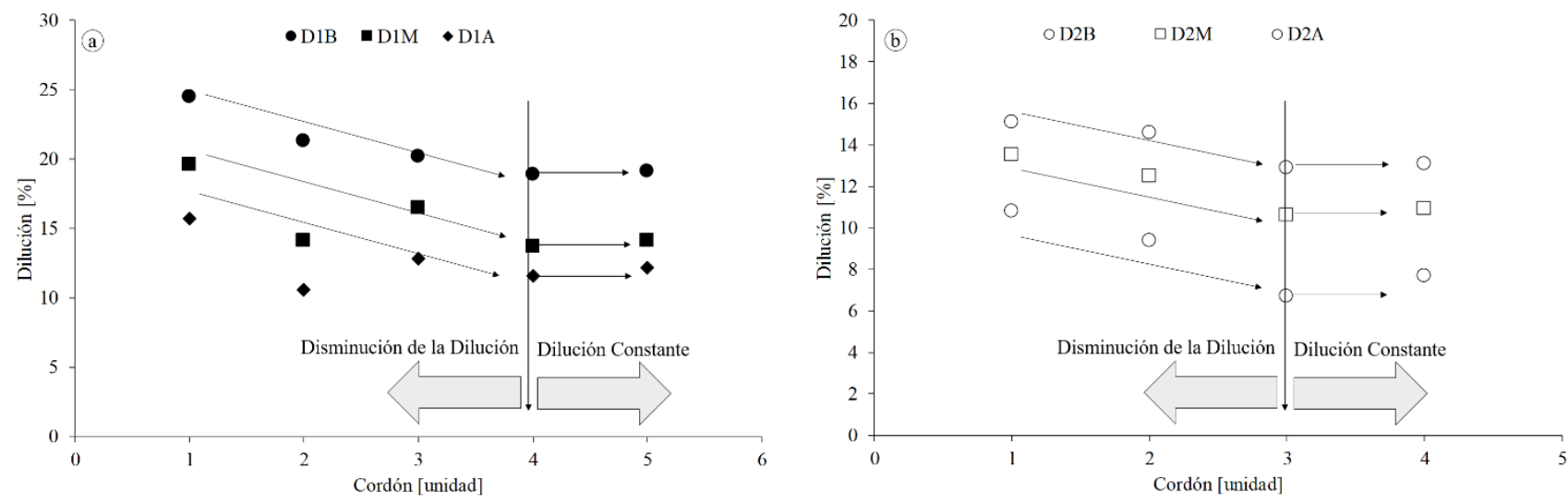

Figura 6. Composición y dilución química.

En la Tabla 4 y Figura 6 se puede observar que, al aumentar el calor aportado se obtiene un leve enriquecimiento de los elementos de aleación (aumentan los contenidos de $\mathrm{Cr}$, $\mathrm{Ni}$ y $\mathrm{Mo}$ ), lo cual genera una disminución de la dilución química, al igual que lo observado anteriormente respecto a la dilución geométrica. Dicho efecto es más evidente en las probetas soldadas con dos capas donde la dilución es baja (para todos los calores aportados) y disminuye de 8 a $2 \%$.

El calor aportado es una medida relativa de la energía transferida por unidad de longitud soldada, siendo un factor de relevancia, ya que afecta el grado de dilución de los recargues soldados y la velocidad de enfriamiento, lo que define la composición química, la evolución microestructural y las propiedades finales del metal depositado.

Por un lado, la dilución es un aspecto de gran importancia en soldadura, fundamentalmente en recubrimientos, donde se buscan bajos niveles de dilución ya que la misma altera la composición química final de los cordones y, por ende, la microestructura y propiedades finales. Cuando la dilución es baja, la composición química del recubrimiento es cercana a la composición química del consumible y las propiedades finales del mismo son las deseadas [8]. Según la literatura [23], al aumentar el calor aportado el contenido de ferrita disminuye en la microestructura. Esto está asociado a que al aumentar el calor aportado disminuye la velocidad de enfriamiento, generando un aumento en el tiempo de transformación de ferrita en austenita, dando como resultado un menor contenido de ferrita en la microestructura. Por otro lado, la literatura [19] menciona que al aumentar los niveles de dilución, el contenido de ferrita disminuye en la microestructura. Un aumento de la dilución provoca un aumento en el contenido de $\mathrm{C}$, sobre todo en materiales consumibles de bajo $\mathrm{C}$, incrementando el contenido de austenita en la microestructura, por el efecto gammágenos del mismo.

Una forma rápida de ordenar a los aceros inoxidables desde el punto de vista de la resistencia a la corrosión localizada es mediante el empleo del PREN (a menor PREN, menor resistencia a la corrosión localizada). En la Tabla 4 se puede observar que, a medida que aumenta el calor aportado, el PREN sube de 28,9 a 33,7 y de 33,1 a 35,2 para una y dos capas respectivamente. 
Es decir, y teniendo en cuenta solamente en PREN (composición química) el cupón de mejor comportamiento a la corrosión localizada sería el D2A (con un PREN de 35,2), asociado a los mayores contenidos de Cr, Mo y N.

\subsection{Diagramas constitucionales}

Acorde con la Figura 7 y, dependiendo del grado de dilución de los cupones soldados, es posible determinar de manera aproximada, la microestructura resultante de los recargues [11,19,22].
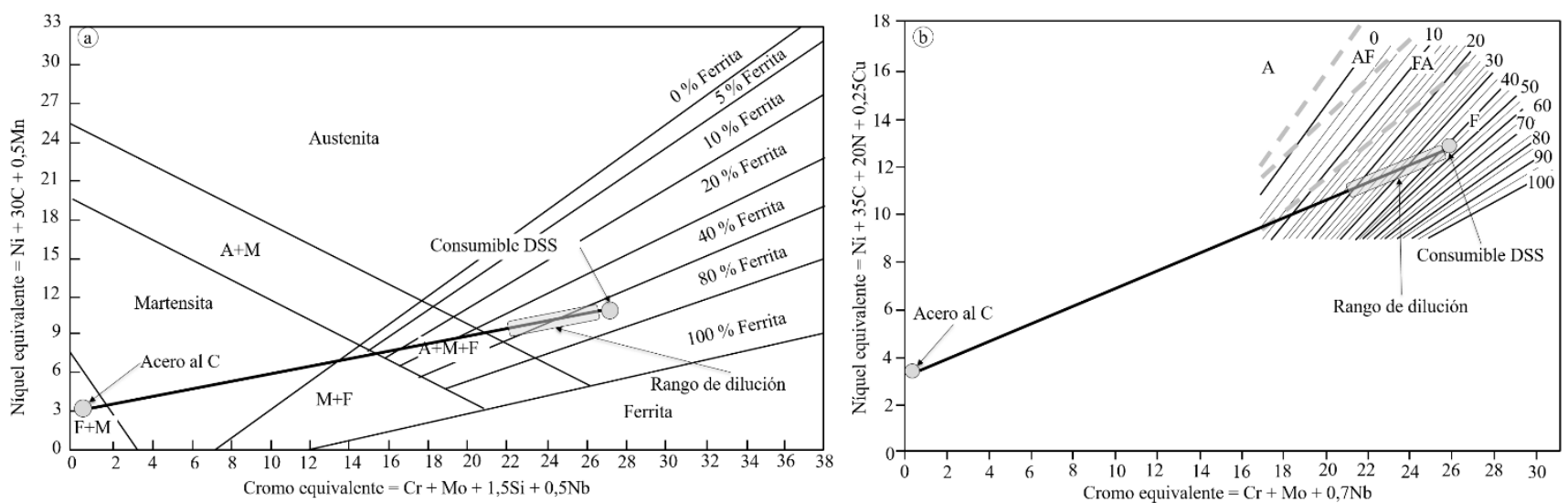

Figura 7. Predicción de la microestructura del recargue: (a) Diagrama Schaeffler; (b) Diagrama WRC 1992.

Los diagramas constitucionales se utilizan para predecir la microestructura del metal de soldadura conociendo la composición química del material base, del material de aporte y la dilución en el metal de soldadura. De acuerdo a lo observado en dichos diagramas de la Figura 7a y b, y en función a la composición química del consumible DSS, sin dilución [16], se puede observar que la microestructura del material de aporte está constituida por un 45 a 60\% de ferrita, para el diagrama WRC1992 y Shaeffler, respectivamente. En cualquiera de los dos diagramas, el fenómeno de dilución disminuiría el contenido de ferrita en el metal de soldadura. Con más de un $20 \%$ de dilución (valor máximo alcanzado en el presente trabajo de investigación) se perdería la característica dúplex del recargue, ya que podríamos entrar en el rango $\mathrm{A}+\mathrm{M}+\mathrm{F}$, situación ya reportada en la literatura [11].

Teniendo en cuenta estos diagramas, la microestructura de los recargues estaría constituida por austenita con un rango de ferrita entre un 30 y 60\%, dependiendo del grado de dilución. A menor dilución, mayor equilibrio microestructural.

Además, la composición química tiene una gran influencia sobre el modo de solidificación y la evolución microestructural. En general, para los aceros inoxidables, dependiendo de la relación $\mathrm{Cr}_{\text {eq }} / \mathrm{Ni}_{\text {eq }}$ es posible determinar el modo de solidificación de acuerdo a las siguientes relaciones [11]:

Modo $A: L \rightarrow L+\gamma \rightarrow \gamma \mathrm{Cr}_{e q} / \mathrm{Ni}_{e q}<1,25$

Modo $A F: L \rightarrow L+\gamma \rightarrow L+\delta+\gamma \rightarrow \delta+\gamma 1,25<C r_{e q} / N i_{e q}<1,48$

Modo FA: $L \rightarrow L+\delta \rightarrow L+\delta+\gamma \rightarrow \delta+\gamma 1,48<C r_{e q} / N i_{e q}<1,95$

Modo $F: L \rightarrow L+\delta \rightarrow \delta \rightarrow \delta+\gamma C r_{e q} / N i_{e q}>1,95$

En este sentido, teniendo en cuenta el grado de dilución obtenido en los recargues (hasta un 20\%) y de acuerdo a la relación $\mathrm{Cr}_{\text {eq }} / \mathrm{Ni}_{\text {eq, }}$ el modo de solidificación obtenido es $\mathrm{F}\left(\mathrm{Cr}_{\text {eq }} / \mathrm{Ni}_{\text {eq }}=2,5\right.$ para todos los casos aproximadamente). Esto es un aspecto relevante, ya que un cambio en el modo de solidificación puede generar variaciones en las microestructuras obtenidas (no solo en cuanto a equilibrio microestructural, sino también a morfología de fases y zonas segregadas) que podrían afectar el comportamiento mecánico y frente a la corrosión.

\subsection{Microscopía óptica y electrónica}

Durante la solidificación de estos aceros, la transformación de ferrita en austenita depende de la composición química y de la velocidad de enfriamiento. Con una alta velocidad de enfriamiento, no hay tiempo suficiente para la transformación de ferrita en austenita, dando como resultado un alto contenido de ferrita. Por el contrario, una baja velocidad de enfriamiento 
otorga mayor tiempo de transformación de ferrita en austenita, generando un mayor contenido de austenita en la microestructura a temperatura ambiente [3]. La secuencia de transformación en estado sólido, durante el enfriamiento, comienza con la nucleación de la Austenita en borde de grano (Abg) en la interfaz de los granos ferríticos ( $\delta / \delta)$, para posteriormente nuclear y crecer la Austenita Widmanstatten (Aw) hacia el interior de los granos. Además de ambas morfologías de austenita, si hay tiempo suficiente para una mayor difusión, puede nuclear y crecer dentro de los granos de ferrita la Austenita Intra Granular (Aig). Como la austenita Abg y la Aw necesitan un menor subenfriamiento, respecto a la austenita Aig, tienen más tiempo para crecer y, por lo tanto, comprenden la mayor parte del contenido final de austenita [22]. Por otro lado, en la zona recalentada de alta temperatura, donde se genera una ferritización parcial, permanecen algunos granos austeníticos. Estos granos austeníticos se denominan generalmente Austenita Parcialmente Transformada (Apt), porque el calentamiento hace que sus elementos de aleación se difundan y, por lo tanto, cambien parcialmente su forma inicial [24]. En este trabajo se identificaron las diferentes morfologías de austenita, mencionadas anteriormente, características de los procesos de soldadura por fusión [5]. La literatura [3] reporta que la zona recristalizada experimenta un recalentamiento que se describe en tres etapas:

1) El material es calentado a muy alta temperatura (sin alcanzar la temperatura de fusión). A esta temperatura, la austenita comienza a transformarse en ferrita hasta que, eventualmente, la estructura es completamente ferrítica. En esta instancia, la mayoría de los posibles precipitados (carburos, nitruros, etc.) comienzan a disolverse;

2) Una vez alcanzada la temperatura pico ocurre crecimiento de grano de la ferrita, ya que no hay segundas fases o precipitados que inhiban su crecimiento;

3) Durante el enfriamiento, la austenita nuclea y crece, mientras que los precipitados se vuelven a formar.

Además, en zonas recalentadas de menores temperaturas los $\mathrm{Cr}_{2} \mathrm{~N}$ son sitios preferenciales para la nucleación de austenita secundaria [3].

La Figura 8 muestra las microestructuras obtenidas tanto por microscopía óptica, como así también por microscopía electrónica de barrido para algunas condiciones de estudio. En todos los casos la microestructura estuvo constituida por dos fases: una clara (austenita) y otra oscura (ferrita). No se encontraron variaciones significativas observables para los diferentes cupones de soldadura, mediante estas técnicas. Sin embargo, en el metal de soldadura, se pudo observar que la microestructura no estuvo balanceada y hubo mayor contenido de austenita en todos los casos. Para los cupones soldados con una capa, al aumentar el calor aportado se observó un leve aumento del contenido de ferrita, asociado a los menores valores de dilución. Por el contrario, para los cupones soldados con dos capas, al aumentar el calor aportado se observó una leve disminución del contenido de ferrita, asociado al mayor tiempo de transformación de ferrita en austenita. Además, la microestructura en la zona afectada por el calor entre cordones (zona recristalizada) estuvo conformada por grandes placas de ferrita con altos contenidos de austenita Secundaria (As) en el interior de las mismas. En la muestra D2B, se puede observar un gran contenido de As y Apt dentro de las placas de ferrita en zona recristalizada entre cordones. En la muestra D2A, se puede apreciar grandes placas de austenita, tanto Abg y Aig. Este incremento del contenido y del tamaño de austenita está asociado al mayor calor aportado y tiempo de transformación de ferrita en austenita.
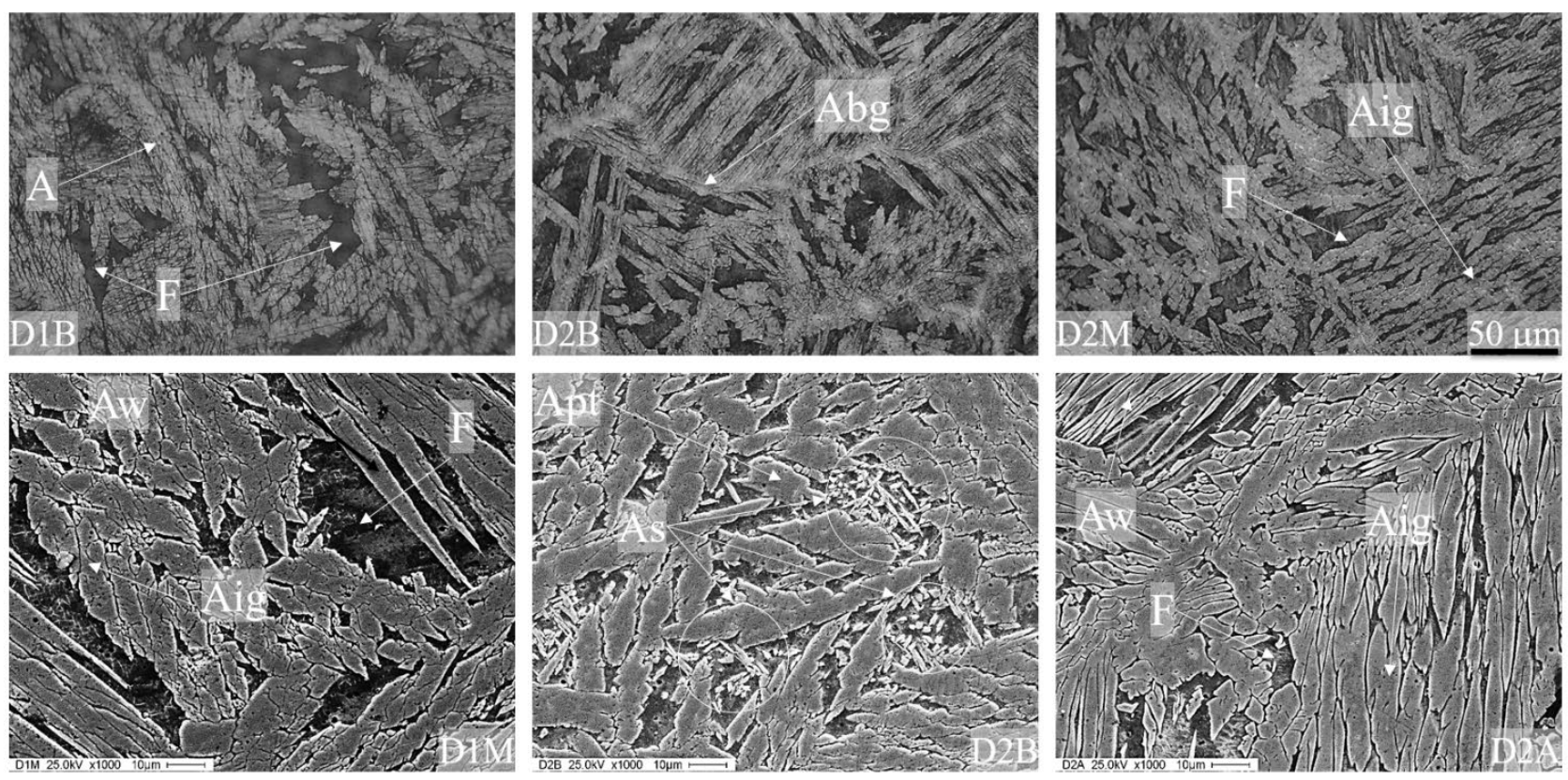

Figura 8. Microscopía óptica y electrónica. 


\subsection{Difracción de rayos $X$}

La fase sigma es una fase que se suele encontrar en muy bajas proporciones dentro del metal de soldadura en los aceros inoxidables dúplex. Para maximizar la identificación de esta fase, se ajustó el rango 2 theta de 42 a $47^{\circ}$, ya que dentro de este intervalo se ubican los picos correspondientes a la fase sigma $[25,26]$. Asimismo, en este rango se observan los picos principales de la fase ferrita y austenita. La Figura 9 muestra los espectros de difracción de rayos X. Dentro del rango 2 theta evaluado, no se ha identificado la presencia de segundas fases. Sin embargo, la técnica de difracción de rayos x tiene un umbral de detección que está en el orden de 4-5\%. Es decir que fases o precipitados presentes por debajo de estos valores no serán detectados mediante dicha técnica.

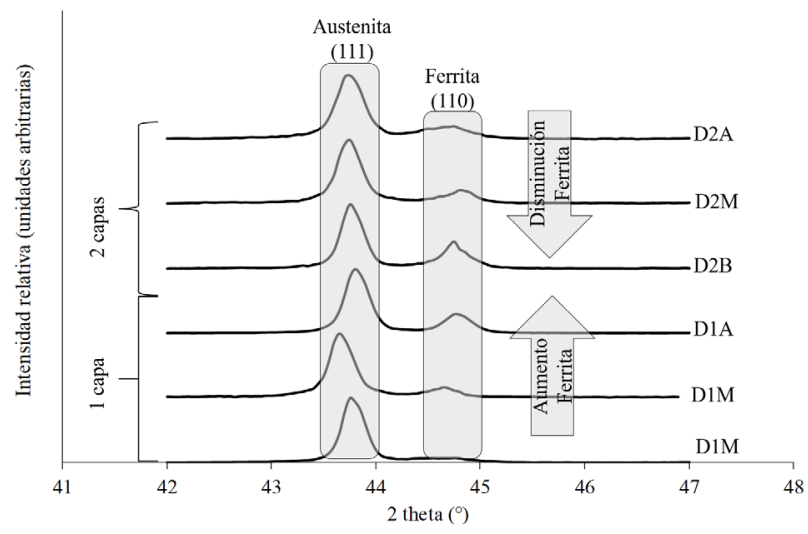

Figura 9. Difractogramas XRD de los cupones soldados.

De acuerdo a la Figura 9, se observan 2 picos correspondientes a la austenita y a la ferrita, con familia de planos (111) y (110), respectivamente. Además, se observa que el pico correspondiente a la austenita es mayoritario en todos los casos, indicando un mayor contenido de la austenita. Además, se aprecia un comportamiento diferente en el pico de ferrita para los cupones de una capa respecto a los de dos capas. Para los cupones soldados con una capa, el pico de ferrita presenta una mayor intensidad, mientras que, para el caso de dos capas, disminuyó al aumentar el calor aportado.

\subsection{Cuantificación microestructural y dureza}

La Figura 10 presenta los contenidos de ferrita, determinados por metalografía color y análisis de imágenes (con un desvío estándar aproximado a 3,5\% para todas las condiciones), y los valores promedio de dureza, medidos sobre la superficie de los recargues para las distintas condiciones de estudio.

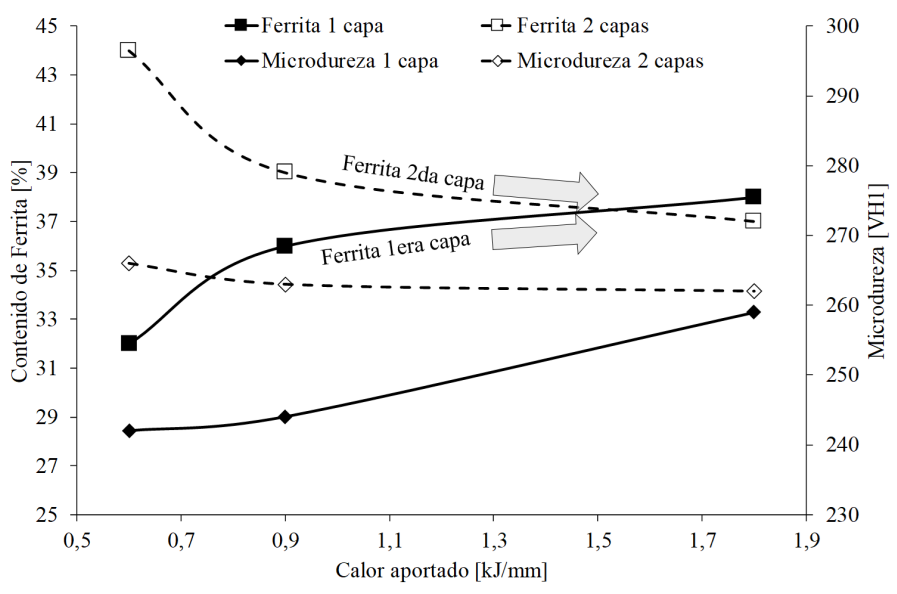

Figura 10. Contenido de ferrita y dureza.

Estas observaciones son consistentes con lo determinado mediante los diagramas constitucionales en el apartado 3.3, a través de los cuales se predijeron fracciones de ferrita semejantes a las obtenidas. Esto pone de manifiesto la validez de la metodología empleada en el presente trabajo a fin de analizar la evolución microestructural y su predicción mediante el uso de estos diagramas. 
De acuerdo con los resultados obtenidos en la cuantificación de ferrita, se presentan dos efectos opuestos para los recargues soldados con una y dos capas. Por un lado, el aumento del calor aportado generó un aumento del contenido de ferrita en los recargues soldados con una capa. Sin embargo, ocurrió el efecto contrario para los recargues soldados con dos capas. Este comportamiento de la evolución del contenido de ferrita al aumentar el calor aportado y la cantidad de capas fue observado en los espectros de difracción de la Figura 9. En este sentido, para los recargues soldados con una capa, el fenómeno de dilución tuvo un mayor efecto sobre el equilibrio microestructural que la velocidad de solidificación [1,8,19], ya que al aumentar el calor aportado disminuyó la dilución generando un incremento en el contenido de ferrita en la microestructura de 32 a $38 \%$. Por otro lado, para los recargues soldados con dos capas, con diluciones bajas y prácticamente constante (entre el 2 y $8 \%$, Tabla 4 ), se puede observar el efecto del calor aportado, ya que el contenido de ferrita disminuyó con el aumento del mismo de 44 al $38 \%$. Un mayor calor aportado genera un mayor tiempo para la transformación de ferrita en austenita durante el enfriamiento, dando como resultado un menor contenido de ferrita en la microestructura $[27,28]$. Ambos fenómenos (dilución y velocidad de enfriamiento) ocurren de manera paralela y tienen influencia en la microestructura final de los recargues. Teniendo en cuenta solamente el equilibrio microestructural, el cupón de mejor comportamiento a la corrosión sería el D2B (con un 44\% de ferrita).

Dicha situación opuesta de la evolución del contenido de ferrita, en los cupones de una capa respecto a los de dos capas es un hecho interesante, ya que al desconocer el efecto de ambas variables (dilución y velocidad de enfriamiento) de manera simultánea se podrían cometer errores en recargues con material de aporte DSS.

Por un lado, un mayor PREN es equivalente a una mayor resistencia a la corrosión por picado [12]. Por otro lado, un mayor equilibrio microestructural de ferrita y austenita, también es sinónimo de una mayor resistencia a la corrosión [15]. Sin embargo, ni el PREN ni el equilibrio microestructural, por si solos, podrían predecir el complejo fenómeno de corrosión. Hay varios efectos microestructurales que favorecen la ruptura de la película pasiva generando el avance de la corrosión localizada como ser: el contenido de inclusiones [14], el tamaño de grano [29], la cantidad de austenita secundaria [13], la precipitación de compuestos [25] e incluso la leve diferencia de composición química entra la ferrita y austenita [30].

Considerando los resultados de dureza para las distintas condiciones de recargue se observa que, si bien no se encontraron grandes variaciones (20 HV1), los recargues soldados con una capa presentaron menores valores de dureza respecto a los recargues soldados con dos capas. Asimismo, en los recargues de una capa, al aumentar el calor aportado aumentó la dureza. Por otro lado, para los recargues soldados con dos capas, la dureza se mantuvo prácticamente constante, mostrando una leve disminución con el aumento del calor aportado. Estos resultados están en concordancia con la cuantificación de ferrita medida en la microestructura para ambos juegos de cupones. De acuerdo a la literatura [31], al aumentar el contenido de ferrita en la microestructura, aumenta la dureza de la misma.

La economía de los recargues, realizados por medio de soldadura por arco eléctrico, esta principalmente controlada por la velocidad de soldadura y la cantidad de capas. En este sentido, la condición D1A, realizada con una capa, presenta una economía de material y de tiempo, obteniendo una dilución relativamente baja (6\%) con una fracción de ferrita de 38\% y un PREN de 33,7. Si bien las condiciones mencionadas anteriormente (D2A y D2B) serían algo superiores en su performance frente a la corrosión localizada, se considera que puede ser una solución que conjuga aspectos técnicos y económicos.

\section{Conclusiones}

A partir del estudio del efecto del aporte de calor y la cantidad da capas en recargues de acero inoxidable dúplex sobre una chapa de acero al carbono se arribó a las siguientes conclusiones:

1) El aumento del calor aportado, a través de la disminución de la velocidad de soldadura, generó un aumento en el volumen del material depositado (alto y ancho) sin tener demasiada influencia en la penetración, dando como resultado una disminución de la dilución para los cupones soldados con una y dos capas;

2) Dentro de una misma capa, la dilución disminuye con las pasadas sucesivas y se hace estable a partir del cuarto cordón, en la primera capa, y del tercer cordón, en la segunda capa. Esto se asocia a disminución de la contribución del material base en el metal fundido;

3) Para el caso de los recargues con dos capas y alto calor aportado se obtuvo la mínima dilución (2\%), lo que generó depósitos con una composición química cercana a la del material de aporte;

4) Se observó un comportamiento diferente para los recargues soldados con una y dos capas. Los cupones soldados con una capa, al aumentar el calor aportado, el contenido de ferrita aumentó (32 a 38\%), debido a la disminución de la dilución. Para los cupones soldados con dos capas (baja dilución), el contenido de ferrita disminuyó (44 a 38\%), asociado a la velocidad de enfriamiento y al mayor tiempo de transformación de ferrita en austenita;

5) La microdureza promedio de los depósitos aumenta con el contenido de ferrita en la microestructura, variado entre 242 y 266 HV. Asimismo, para contenidos de ferrita similares, los recargues de dos capas presentaron mayores valores de microdureza, lo que se asociaría al mayor contenido de aleación; 
6) Las condiciones con dos capas presentaron mayores valores PREN y de ferrita. La condición con una capa y alto aporte térmico (D1A) presentó valores semejantes, aunque algo menores, constituyéndose en una alternativa tecnológica interesante, aumentando la productividad y disminuyendo los costos.

\section{Agradecimientos}

Los autores agradecen a Daniel Betz de BOHELER Argentina por la provisión del consumible utilizado, a Ricardo Flores de AIR LIQUIDE Argentina por la donación de los gases de soldadura, a Eduardo Asta de ESAB-CONARCO Argentina por los análisis químicos, a Mercedes Pianetti del Laboratorio de Microscopía Electrónica del INTI - MECÁNICA por las imágenes SEM, a Silvana Arroyo de INTECIN por los espectros de Difracción de Rayos X y a la UNLZ por el aporte financiero.

\section{Referencias}

[1] Kannan T, Murugan N. Effect of flux cored arc welding process parameters on duplex stainless steel clad quality. Journal of Materials Processing Technology. 2006;176(1-3):230-239. http://dx.doi.org/10.1016/j.jmatprotec.2006.03.157.

[2] Zappa S, Zalazar M, Surian E. Efecto de la composición química del metal de aporte y del calor aportado sobre la microestructura y las propiedades mecánicas de juntas soldadas de aceros inoxidables dúplex. Soldagem e Inspeção. 2017;22(2):116-128. http://dx.doi.org/10.1590/0104-9224/si2202.02.

[3] Lippold JC, Kotecki DJ. Welding metallurgy and weldability of stainless steels. USA: Wiley-Interscience; 2005. $357 \mathrm{p}$

[4] Villalobos D, Maldonado C, Albiter A, Robles-Piedras E. Efecto de los ciclos térmicos sobre la ZAT de una soldadura multipasos de un acero inoxidable superdúplex SAF 2507. Soldagem e Inspeção. 2010;15(3):170-176. http://dx.doi.org/10.1590/S010492242010000300002.

[5] Eghlimi A, Ra K, Shamanian M. Tribocorrosion behavior of overlay welded super duplex stainless steel in chloride medium. Journal of Bio Tribo Corros. 2015;2015(3):1-18. http://dx.doi.org/10.1007/s40735-015-0018-8.

[6] Senthilkumar B, Kannan T. Sensitivity analysis of flux cored arc welding process variables in super duplex stainless steel claddings. Procedia Engineering. 2013;64:1030-1039. http://dx.doi.org/10.1016/j.proeng.2013.09.180.

[7] Senthilkumar B, Kannan T. Effect of flux cored arc welding process parameters on bead geometry in super duplex stainless steel claddings. Measurement. 2015;62:127-136. http://dx.doi.org/10.1016/j.measurement.2014.11.007.

[8] Kannan T, Murugan N. Prediction of ferrite number of duplex stainless steel clad metals using RSM. Welding Journal. 2006;85(91):91s100 s.

[9] Eghlimi A, Shamanian M, Raeissi K. Dilution and ferrite number prediction in pulsed current cladding of super-duplex stainless steel using RSM. Journal of Materials Engineering and Performance. 2013;22(12):3657-3664. http://dx.doi.org/10.1007/s11665-013-0661-5.

[10] Luchtenberg P, de Campos PT, Soares P, Laurindo CAH, Maranho O, Torres RD. Effect of welding energy on the corrosion and tribological properties of duplex stainless steel weld overlay deposited by GMAW/CMT process. Surface and Coatings Technology. 2019;375:688693. http://dx.doi.org/10.1016/j.surfcoat.2019.07.072.

[11] Di X, Zhong Z, Deng C, Wang D, Guo X. Microstructural evolution of transition zone of clad X70 with duplex stainless steel. Materials \& Design. 2016;95:231-236. http://dx.doi.org/10.1016/j.matdes.2016.01.087.

[12] Zhang Z, Jing H, Xu L, Han Y, Zhao L, Zhou C. Effects of nitrogen in shielding gas on microstructure evolution and localized corrosion behavior of duplex stainless steel welding joint. Applied Surface Science. 2017;404:110-128. http://dx.doi.org/10.1016/j.apsusc.2017.01.252.

[13] Zhang Z, Jing H, Xu L, Han Y, Zhao L, Zhang J. Influence of microstructure and elemental partitioning on pitting corrosion resistance of duplex stainless steel welding joints. Applied Surface Science. 2017;394:27-314. http://dx.doi.org/10.1016/j.apsusc.2017.03.179.

[14] An L, Cao J, Wu L, Mao H, Yang Y. Effects of Mo and Mn on pitting behavior of duplex stainless steel. Journal of Iron and Steel Research International. 2016;23(12):1333-1341. http://dx.doi.org/10.1016/S1006-706X(16)30196-0.

[15] López MEH, Delgado EH, Torres DT, Morquecho AMA. Identificación de fases y precipitados por MFA en uniones de acero inoxidable dúplex. soldagem e Inspeção. 2016;21(2):237-250. http://dx.doi.org/10.1590/0104-9224/SI2102.13.

[16] Böhler. Technical Information: BÖHLER CN 22/9 PW-FD: GMAW flux cored wire, high-alloyed, highly corrosion resistant. Düsseldorf; 2007.

[17] Eghlimi A, Shamanian M, Raeissi K. Effect of current type on microstructure and corrosion resistance of super duplex stainless steel cladding produced by the gas tungsten arc welding process. Surface and Coatings Technology. 2014;244:45-51. http://dx.doi.org/10.1016/j.surfcoat.2014.01.047.

[18] Dupont JN, Kusko CS. Technical note: martensite formation in austenitic/ferritic dissimilar alloy welds. Welding Journal. 2007;86:51-54.

[19] Kotecki DJ. Dilution control in single-wire stainless steel submerged arc cladding. Welding Journal. 1996;75(2):35s-45s. 
[20] Shamanian M, Eghlimi A, Eskandarian M, Szpunar JA. Interface microstructure across cladding of super duplex stainless steel with austenitic stainless steel buffer layer. Surface and Coatings Technology. 2014;259:532-542. http://dx.doi.org/10.1016/j.surfcoat.2014.10.034

[21] Eghlimi A, Shamanian M, Eskandarian M, Zabolian A, Nezakat M, Szpunar JA. Evaluation of microstructure and texture across the welded interface of super duplex stainless steel and high strength low alloy steel. Surface and Coatings Technology. 2015;264(25):150-162. http://dx.doi.org/10.1016/j.surfcoat.2014.12.060.

[22] Rahmani M, Eghlimi A, Shamanian M. Evaluation of microstructure and mechanical properties in dissimilar austenitic/super duplex stainless steel joint. Journal of Materials Engineering and Performance. 2014;23(10):3745-3753. http://dx.doi.org/10.1007/s11665-0141136-z.

[23] Almenara MD, et al. Modelos empíricos para la predicción de la geometría del cordón en soldaduras a tope de un acero inoxidable dúplex 2205. Dyna. 2011;78(169):206-215.

[24] Eghlimi A, Shamanian M, Eskandarian M, Zabolian A, Szpunar JA. Characterization of microstructure and texture across dissimilar super duplex/austenitic stainless steel weldment joint by austenitic filler metal. Materials Characterization. 2015;106:208-217. http://dx.doi.org/10.1016/j.matchar.2015.05.036.

[25] Del Abra-Arzola JL, García-Rentería MA, Cruz-Hernández VL, García-Guerra J, Martínez-Landeros VH, Falcón-Franco LA, et al. Study of the effect of sigma phase precipitation on the sliding wear and corrosion behaviour of duplex stainless steel AISI 2205. Wear. 2018;400401:43-51. http://dx.doi.org/10.1016/j.wear.2017.12.019.

[26] Garin JL, Mannheim RL, Camus MA. Formación de fase sigma en uniones soldadas de acero inoxidable súper dúplex fundido. Revista de Metalurgia. 2011;47(4):293-306. http://dx.doi.org/10.3989/revmetalm.1041.

[27] Vitec JM, Davis SA, Hinman CR. Improved ferrite number prediction model that accounts for cooling rate effects. Part 1: model development. Welding Journal. 2003;82(1):10-17.

[28] Vitec JM, Davis SA, Hinman CR. Improved ferrite number prediction model that accounts for cooling rate effects. Part 2: model results. Welding Journal. 2003;82(2):43-50.

[29] Wan J, Lou Y, Ruan $\mathrm{H}$. The partition coefficient of alloying elements and its influence on the pitting corrosion resistance of $15 \mathrm{Cr}-2 \mathrm{Ni}$ duplex stainless steel. Corrosion Science. 2018;139:13-20. http://dx.doi.org/10.1016/j.corsci.2018.04.038.

[30] Kang DH, Lee HW. Study of the correlation between pitting corrosion and the component ratio of the dual phase in duplex stainless steel welds. Corrosion Science. 2013;74:396-407. http://dx.doi.org/10.1016/j.corsci.2013.04.033.

[31] Martins M, Casteletti LC. Effect of heat treatment on the mechanical properties of ASTM A 890 Gr6A super duplex stainless steel. Journal of ASTM International. 2005;2(1):1-14. 